\title{
Static and dynamic factors in the perception of rotary motion
}

\author{
MYRON L. BRAUNSTEIN and KENNETH R. STERN \\ University of California, Irvine, California 92717
}

\begin{abstract}
Direction of rotation and static slant judgments were collected for a series of outline plane forms in four experiments with 106 subjects. Direction was judged more accurately for forms displaying the same perspective gradients as trapezoids, but with right-angled contours, than for trapezoids. There were no consistent differences among these forms in judged slant. Direction of rotation judgments were affected by a static false interposition cue, with interposition increasing the proportion of veridical judgments when placed in conflict with a relative size gradient and decreasing this proportion when a size gradient was absent. Both a dynamic factor (contour angle change) and a static factor (misperceived relative distance of the vertical sides) were found to affect perceived direction of rotation, with direction judgments based primarily on the dynamic factor.
\end{abstract}

Most research on the perception of the direction of rotation of two-dimensional forms has concentrated on the misperception of direction for trapezoids or for forms that share certain geometric characteristics with trapezoids. As the viewing distance from a trapezoid is increased (Braunstein \& Payne, 1968) and as the difference between the vertical sides is increased (Braden, 1978), the perception of direction of rotation becomes divisible into two phases: a veridical phase which corresponds roughly to the period when the larger vertical side is closer, and an illusory phase which occurs when the larger side is more distant but appears closer. This perceived oscillation of a rotating trapezoid provides evidence against three hypotheses that might otherwise be accepted as general principles of perception: (1) A rigid object is perceived whenever there is a rigid object that geometrically could be the cause of the proximal stimulus. (There is no oscillating rigid object that could produce the proximal changes projected by a rotating trapezoid.) (2) Ambiguous proximal stimulation is resolved by selecting one (or more) geometrically possible distal object motions as the percept. (An oscillating plane is not one of the geometric possibilities.) (3) An increase in the visual angle subtended by a part of a form implies that the part is approaching. (In the illusory phase, the smaller side may increase in visual angle and be perceived as receding, even for a magnitude of increase that can be shown to be detectable.)

Explanations of the misperception of direction of rotation of a trapezoid may be classified as emphasizing either static characteristics of the trapezoidal

This research was supported by National Science Foundation Grant BNS-76-81499. The design of the stimulus forms was based on suggestions by Dr. Walter Gogel and Dr. John Yellott, to whom the authors are indebted. Duane Dike, Jack Schryver, and Robert Theal assisted in running the subjects. form or dynamic characteristics of the changing projection of the form as the trapezoid rotates. Ames (1951) proposed that misperception of direction occurred when the larger side was more distant because the trapezoid was perceived as a rectangular window and the side with the larger retinal projection therefore always appeared closer. A number of similar explanations have been advanced based on linear perspective and relative size-static characteristics of the trapezoid. The principal source of evidence for these explanations is the correlation found between judgments of the slant angle of trapezoids (and other figures made to oscillate like trapezoids) with the frequency of oscillation or with the apparent point of oscillation (Olson, 1974; Power \& Day, 1973).

Braunstein (1972) proposed an explanation of the perception of direction of rotation of rectangles and trapezoids based on dynamic characteristics of the two-dimensional projections of the rotating figures. According to this explanation, direction judgments are determined primarily by changes in the projected contour angles, if these changes are detectable. An increase in the magnitude of the angles enclosing a vertical side is associated with perceived recession of that side; a decrease in the angles is associated with perceived approach. Geometrically, an increase in the projected angles enclosing a vertical side of a rectangle is always the result of recession of the enclosed side and a decrease is always the result of approach. For a sufficiently distant trapezoid, however, the angles enclosing the larger side will decrease and the angles enclosing the smaller side will increase whenever the figure leaves the frontal plane, regardless of direction of rotation. In parallel projections of rotating rectangles and trapezoids (simulating an infinite viewing distance with respect to perspective effects), the projected angles in the rectangle do not 
change during rotation, but the angles enclosing the larger side of a trapezoid still decrease and the angles enclosing the smaller side still increase as the figure leaves the frontal plane. Direction of rotation judgments approach chance for rectangles and approach fully predictable cycles of oscillation for trapezoids as the viewing distance increases (Braunstein \& Payne, 1968).

It is difficult to distinguish experimentally between explanations of misperceived direction of rotation of trapezoids based on misperceived static orientation and explanations based on dynamic contour angle changes, because the same characteristics of a form, such as the ratio of the vertical sides (for a fixed width), often determine both judged slant and the contour angle change function. There are two related types of evidence, however, that would be useful in supporting a contour angle explanation. First, if misperception of rotation could be reduced by substituting right angles for acute and obtuse angles in a form, while leaving the vertical sides unequal, then misperceived direction judgments could not be based simply on misperceived relative distance of the vertical sides caused by their relative sizes. The second type of evidence would be a demonstration that there are stimulus manipulations that reduce misperception of rotation without reducing perceived static slant. Evidence of the first type has been provided by Börjesson (1971) and Braunstein (1971), using various forms in which unequal sides were associated with right angles (or equal sides were associated with acute and obtuse angles). (See Figure 1.) It can be argued that these studies did not adequately test static slant explanations of the trapezoid illusion, as the presence of two unequal vertical sides in these forms may not have been as effective as the continuous size gradient present in the Ames trapezoid. The question of whether or not forms with right angles and unequal sides appeared less slanted than trapezoids was not addressed in these studies. Evidence of the second type, that misperception of rotation can be reduced by figural changes that do not reduce judged slant, is not currently available.

The present study considers both types of evidence for the contour angle explanation by introducing forms that are trapezoidal in overall shape but are constructed with rectangular segments (gradedrectangle forms). Both direction of rotation judgments and slant judgments are collected for these forms, as well as for rectangular and trapezoidal control forms.

The present study also considers evidence that would favor a misperceived static slant explanation of misperception of direction of rotation over a dynamic contour angle change explanation. Appropriate evidence would be a demonstration that perceived direction of rotation can be affected by a

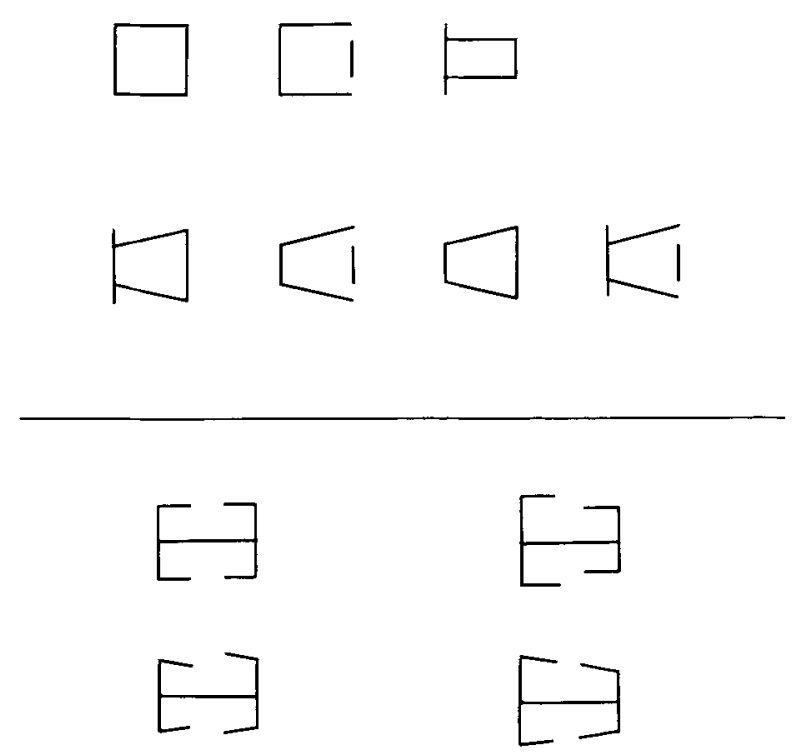

Figure 1. Examples of forms studied by Börjesson (1971) (top) and Braunstein (1971) (bottom).

figural characteristic related to perceived relative distance of the sides in the static form but not related to angle change. Evidence of this type is found in a study by Cross and Cross (1969) in which the addition of false shadows increased reported oscillations even for a rotating rectangle. Experiments 1 and 2 include forms in which contour interruption is used to suggest that the rectangular segments comprising the form vary in distance. The apparent interposition of these segments does not vary during rotation, as the form is actually a plane figure with contour interruptions.

\section{EXPERIMENT 1}

\section{Method}

Subjects. The subjects were 41 students in lower division psychology courses at the University of California, Irvine, who received extra credit for their participation. Vision of at least 20/40 (Snellen eye chart) was required in the eye used in the experiment. Data from one subject, who did not meet the check trial criterion (see Procedure), were not used in the analysis.

Stimuli. The stimuli were 12 plane outline forms rotating about a vertical axis through the center of the form. The forms were generated (computationally) by rotating an outline rectangle 2.56 units wide $\times .90$ units high about its center to an angle of $63.4 \mathrm{deg}$ and projecting it onto a plane through the origin, using a projection point 6 units from the origin. The resulting trapezoid is the outline of form $c$ in Figure 2. The graded-rectangle forms were produced by placing five smaller rectangles at equal distances along the slanted rectangle. These smaller rectangles were equal in size and frontally oriented. The width of the rectangles was selected to produce a $25 \%$ horizontal overlap. Form $b$ is the projection of these five segments, with hidden lines deleted. Form a was produced by starting with the same five rectangles as in form $b$, but deleting portions of the contours as if the more distant rectangles were the closer ones, thus placing interposition in conflict with the relative size gradient. Vertical lines were added to the 


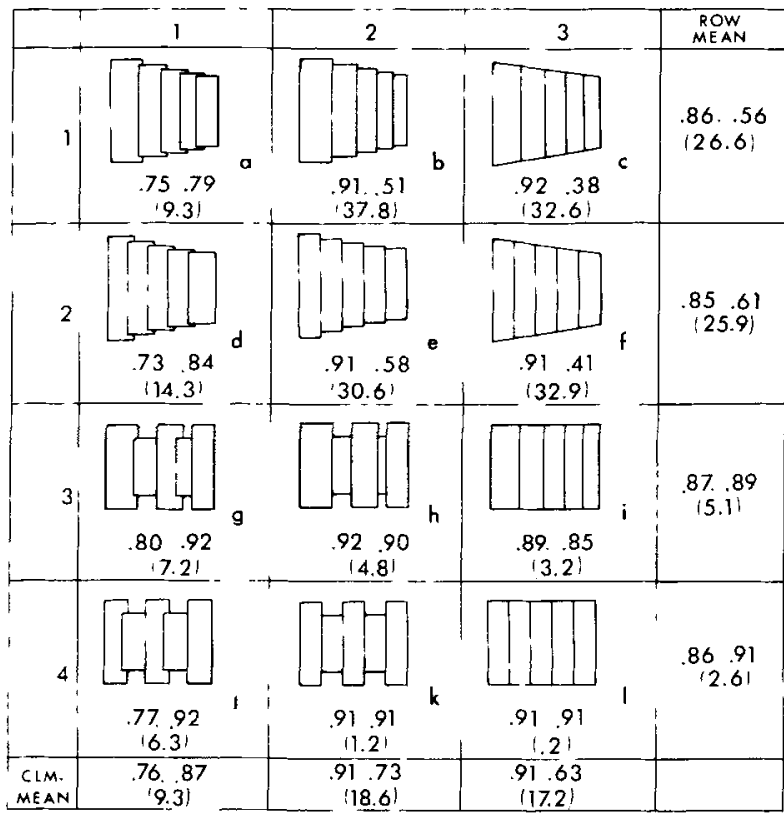

Figure 2. Stimuli and results for Experiments 1 and 2. Results are shown as $\mathrm{T} 1, \mathrm{~T} 2(\mathrm{~S})$, where $\mathrm{T} 1$ and $\mathrm{T} 2$ are the mean proportions of time that direction of rotation was judged correctly for Phase $I$ and Phase II (Experiment 1$)$ and $S$ is the mean judged slant (Experiment 2).

trapezoid in form $\mathrm{c}$ to produce a horizontal gradient matching the horizontal perspective gradient in form $b$. The remaining forms were produced by eliminating the horizontal perspective gradient (row 2), the vertical perspective gradient (row 3), or both perspective gradients (row 4). In rows 3 and 4 , the heights of the second and fourth segments were reduced to allow a display of overlap in the absence of a vertical perspective gradient.

All forms were 1.2 units wide. The vertical sides of the forms in rows 1 and 2 were 1.12 units and .76 units, respectively. The outer vertical sides in rows 3 and 4 were .90 units. Each stimulus sequence displayed 1 of the 12 forms rotating twice through $360 \mathrm{deg}$, and projected onto a plane from a projection distance of $3,6,12,24$, or 48 units from the origin. The rotation speed was $8 \mathrm{rpm}$. A sequence lasted $15 \mathrm{sec}$, with a 5 - sec ISI. Direction of rotation was counterbalanced across figures, perspective levels, and subjects.

Apparatus. The displays were presented on a CRT (VR-17), which was part of a GT-42 graphics system under the control of a PDP-11/40 minicomputer (all manufactured by the Digital Equipment Corporation). The subject viewed the displays monocularly through a lens system that projected a real image optically located approximately $1 \mathrm{~m}$ in front of the subject's eye. The lens system consisted of a $50-\mathrm{mm}$ enlarging lens, used to project the VR-17 image onto a smaller rear-projection screen, and a 50-mm camera lens between the rear-projection screen and the eye. The focal point of the camera lens was $52 \mathrm{~mm}$ from the rear-projection screen. A .8 neutral density filter and a No. 21 Wratten filter were mounted in front of the rear-projection screen to eliminate any visible phosphor trace and to change the hue of the forms from green to a more achromatic appearance. The stimulus forms appeared as light figures (approximately $.01 \mathrm{fL}$ ) against a dark background (less than $.0005 \mathrm{fL}$ ). A 9.5 -mm-diam aperture at the subject's eye limited the field of view to a circular area 18.9 deg in diameter. One unit in the display computations was equivalent to 9.46 deg in visual angle. The subject's viewing distance was thus equivalent to a projection distance of 6 units in the display computations.
The response device was a wooden plane, $80 \mathrm{~mm}$ wide $\times 60 \mathrm{~mm}$ high, located on a table below the viewing aperture. Turning the plane clockwise or counterclockwise actuated one of two microswitches in a circuit between two oscillators and an audio tape recorder, producing a continuous record of the subject's response on audio tape. The tapes were later converted to digital form for computer analysis. A demonstration device consisting of a $150-\mathrm{mm}$ wide $\times 115$-mm-high rectangle mounted on an 8-rpm reversible motor was used in instructing the subjects.

Procedure. The subjects were told that they would be viewing a series of figures that would be rotating clockwise, rotating counterclockwise, or oscillating. They were instructed to turn the response plane in the same direction as that of the figure and to change their response whenever the figure appeared to change direction. They were told to indicate one direction or the other at all times while the display was visible. The subjects practiced the response procedure, with the demonstration device serving as the stimulus. The room was then made totally dark and the displays were presented.

A total of 68 stimulus sequences were presented to each subject. The first 4 were practice trials, and the last 4 were high-perspective stimuli used to check for any confusion in the use of the response device. Data for subjects responding incorrectly to more than one of the four check trials were not used in the analysis. Order of presentation of the 60 regular stimuli was randomized separately for each subject.

\section{Results and Discussion}

The proportion of time that the direction of rotation reported by a subject matched the veridical direction was determined for each stimulus. Only the second $360 \mathrm{deg}$ of rotation was used in the analysis, and this 360 deg was divided into two 180-deg phases: In Phase 1 , the side of the form shown at the left in Figure 2 was closer to the observer's viewpoint (in the display computations). This would be the large side for the trapezoidal shapes, and Phase I therefore corresponds to the veridical phase in the rotating trapezoid illusion. In Phase II, the side shown at the left was the more distant side. Phase II corresponds to the illusory phase in the rotating trapezoid illusion. Whether a given subject responded first to Phase I or Phase II for a particular stimulus depended on the direction of rotation, which was counterbalanced. The mean proportions of veridical response time are shown in Figure 2. Separate fourway analyses of variance (gradients, overlap, perspective, and subjects) were conducted for the Phase I and Phase II proportions.

Phase I. Significant main effects were found for overlap $[F(2,78)=54.2, p<.01]$ and for perspective $[F(4,156)=122.2, p<.01]$. Post hoc comparisons (Tukey HSD test; Lee, 1975) showed the overlap effect to be due to a significantly lower proportion of veridical response time for the forms showing explicit interposition of segments (column 1 in Figure 2) than for those not showing explicit interposition (columns 2 and 3). Interposition in the column 1 forms suggests that the side on the left is more distant, but in Phase I of the rotation cycle, the side on the left is the closer side. This suggests that an incorrect perception of the relative distances of the vertical sides 
may account for the lower proportion of veridical response time found for the forms showing explicit interposition.

A higher proportion of veridical judgment time was found for closer projection distances, as expected from previous research (Braunstein, 1971; Braunstein \& Payne, 1968; Petersik, 1979). The main effect of gradients was not significant $[F(3,117)<1]$. There were significant interactions of perspective with overlap $[F(8,312)=18.9, p<.01]$ and perspective with gradient $[\mathrm{F}(12,468)=3.53, \mathrm{p}<.01]$. The effects of overlap and gradient increased with increasing projection distances (see Figure 3 ).

Phase II. Significant main effects $(p<.01)$ were found for overlap $[F(2,78)=94.2]$, gradient $[F(3,117)=$ 283.6], and perspective $[F(4,156)=235.0]$. Post hoc comparisons for gradients showed significantly lower proportions of veridical response time for stimuli displaying vertical gradients (rows 1 and 2 in Figure 2) than for stimuli displaying either horizontal gradients (row 3) or no gradients (row 4). There were no sig-

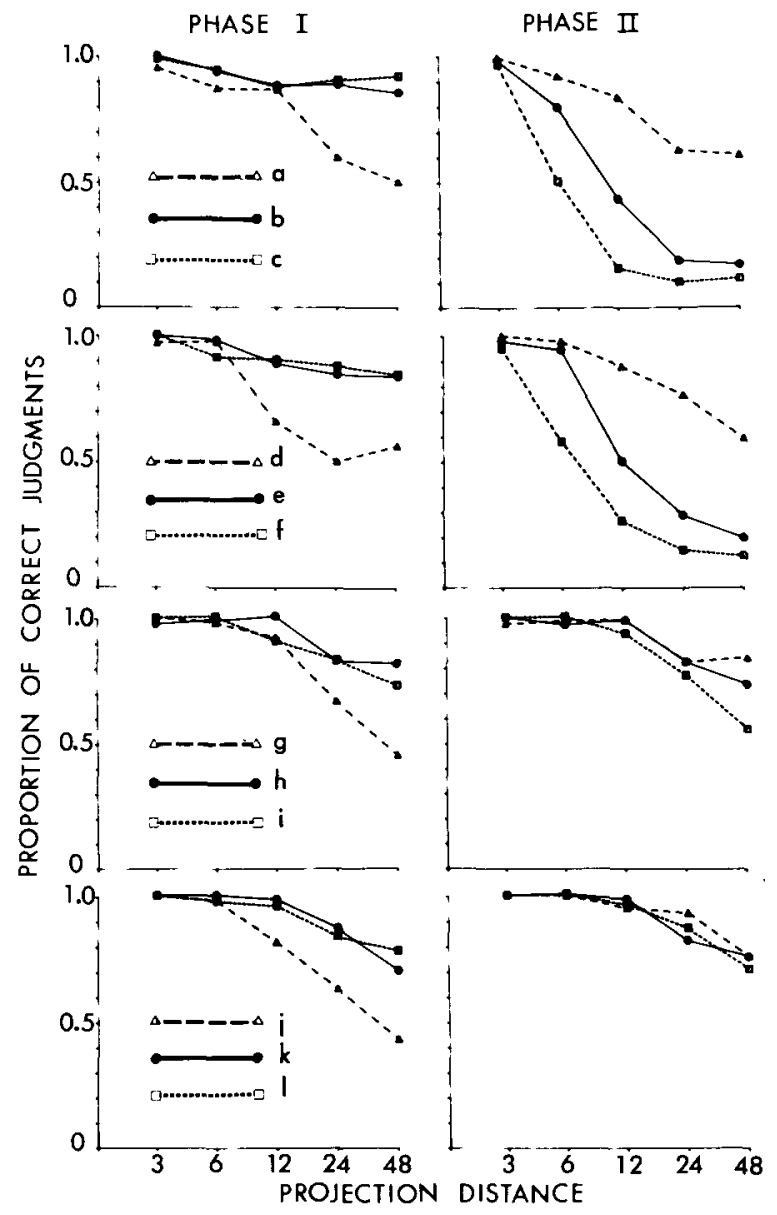

Figure 3. Overlap by perspective interactions for each gradient level, for Phase I (left) and Phase II (right). (Small letters refer to forms in Figure 2.) nificant differences attributable to horizontal gradients. This confirms previous findings (Braunstein, 1977; Braunstein \& Payne, 1968; Hershberger, Stewart, \& Laughlin, 1976) that direction of rotation is determined primarily by effects in the dimension of the axis of rotation, the vertical dimension in this experiment.

The interaction of overlap and gradient $[F(6,234)=$ 44.2] was significant $(\mathrm{p}<.01)$. Post hoc comparisons were run among forms $\mathrm{a}, \mathrm{b}$, and $\mathrm{c}$ and among forms $\mathrm{d}$, e, and $\mathrm{f}$ to determine whether the rotating trapezoid illusion was reduced by the use of rightangled segments and by placing interposition in conflict with the vertical perspective gradient. Direction judgments for form $b$ were significantly more veridical than for form c. These forms have the same relative size gradients, indicating that the presence of right angles can increase accuracy when the relative size gradient is held constant. This was confirmed in the absence of horizontal gradients by a similar significant difference between forms e and f. Conflicting interposition information further reduced the nonveridical judgment time, as evidenced by significant differences between forms $a$ and $b$ and between $\mathrm{d}$ and $\mathrm{e}$.

As in Phase I and previous research, a higher percentage of veridical judgment time was found with decreasing projection distance. Perspective interacted significantly $(\mathrm{p}<.01)$ with overlap $[\mathrm{F}(8,312)=$ 14.7] and with gradients $[F(12,468)=26.9]$. Overlap and gradient differences increased with increasing projection distance (Figure 3). The interaction of the three independent variables was also significant $[F(24,936)=3.86, p<.01]$.

\section{EXPERIMENT 2}

The purpose of Experiment 2 was to collect static slant judgments for the 12 forms studied in Experiment 1 .

\section{Method}

Subjects. Eighteen paid university students served as subjects. The same vision criterion was employed as in Experiment 1.

Stimuli. The stimuli were $35-\mathrm{mm}$ black-and-white slides of the 12 forms in Experiment 1.

Apparatus. The slides were projected onto a Polacoat rearprojection screen and were viewed monocularly from a distance of $1.8 \mathrm{~m}$ through a circular aperture of the same diameter as in Experiment 1. Neutral density filters were used to equate the brightness of the forms with the brightness levels recorded in Experiment 1. The subject's response device was a plywood rectangle $150 \mathrm{~mm}$ wide $\times 115 \mathrm{~mm}$ high $\times 19 \mathrm{~mm}$ thick, painted flat black with a 19-mm-wide white border. The rectangle was attached to both a reversible motor for returning it to a frontal position and a shaft encoder that displayed its slant digitally to an accuracy of $1 \mathrm{deg}$, at the experimenter's station. A grid of white lines was mounted just below the response plane to provide maximum information to the subject about the slant of the response plane.

Procedure. The subjects were told that they would be viewing a 
series of figures that might be slanted with either the left or the right side closer to them, and that after each display they were to move the response plane to the apparent slant of the figure seen through the viewing tube. Each slide was presented for $15 \mathrm{sec}$, after which the response plane was illuminated. The subject moved the response plane to the desired slant and pressed a button to indicate that a judgment was completed. The lights then were turned off and the next slide was presented. The response plane was returned to a frontal orientation while the subject viewed the next display. A total of 40 slides were presented, consisting of 4 practice slides and three presentations of each of the 12 forms, with order of presentation randomized separately for each subject.

\section{Results and Discussion}

Mean judged slants for the 12 stimuli are shown in Figure 2. The main effects of overlap $[F(3,51)=60.3]$ and of gradient $[F(2,34=54.6]$, and their interaction $[F(6,102)=32.6]$, were significant $(p<.01)$. Post hoc comparisons (Tukey HSD tests) showed forms displaying both vertical and horizontal gradients or vertical gradients only (rows 1 and 2 in Figure 2) to differ significantly in judged slant from those displaying horizontal gradients only or no gradients (rows 3 and 4). Post hoc comparisons for column effects within rows showed significantly lower slant judgments for forms displaying conflicting interposition and vertical gradient information than for forms that did not display this conflicting information ( $a$ vs. $b$ and $c$ in row 1 and $d$ vs. $e$ and $f$ in row 2 ). The differences between forms $b$ and $c$ and between $e$ and $f$ were not significant, nor were any column effects in rows 3 and 4.

The most important finding was the lack of a systematic relationship between judged slant in this experiment and the proportion of time that direction was judged veridically in Experiment 1 , for forms $b$, $c, e$, and $f$. If misperceived static slant were the cause of nonveridical direction of rotation judgments, larger slant judgments should have been associated with smaller proportions of veridical judgment time. In Experiment 1, the proportion of Phase II veridical judgment time was significantly greater for $b$ than for $c$ and for e than for $f$, but these forms did not differ significantly in judged slant in the present experiment. (For $b$ and $c$, the obtained slant difference was in the direction opposite to that predicted by a misperceived static slant explanation of misperceived rotary motion.) These results suggest that variations in the misperception of the direction of rotary motion cannot be attributed to variations in misperceived static slant when comparisons are made among forms differing in the presence or absence of right angles, but not in overall shape. Stimulus variables related to overall shape may affect both static slant judgments and direction of rotation judgments (Olson, 1974; Power \& Day, 1973), but a causal relationship should not be inferred between these two perceptions.

\section{EXPERIMENT 3}

Two variations in the graded-rectangle form are introduced in Experiment 3, in order to test the generality of the finding of a higher proportion of veridical judgment time when right angles are included in forms having perspective gradients matching those of trapezoids. These variations in the internal structure of the forms were intended to vary the effectiveness of the relative size gradient as an indicator of relative distance. By eliminating the internal divisions of form a in Figure 4, producing form $b$, a reduction in the perceived relative distances of the outer vertical contours of the form might be expected. The new form should appear to be a single plane form, rather than a series of five forms that could be located at varying distances in depth. On the other hand, elimination of alternate segments of form a (Figure 4), producing form $\mathrm{c}$, should increase perceived depth, as the separation of the remaining rectangles would reduce the tendency to perceive the rectangles as being at the same distance, due to an adjacency effect (Gogel, 1970).

A second purpose of Experiment 3 was to eliminate the suggested overlap in the graded-rectangle forms that may have resulted from the horizontal gradient (see form b in Figure 2). This gradient was based on the projection of overlapping rectangles in Experiments 1 and 2. A gradient based on adjacent rectangles is used in Experiments 3 and 4.

\section{Method}

Subjects. The subjects were 30 students from the same source as in Experiment 1. The vision criterion was the same as in Experiment 1.

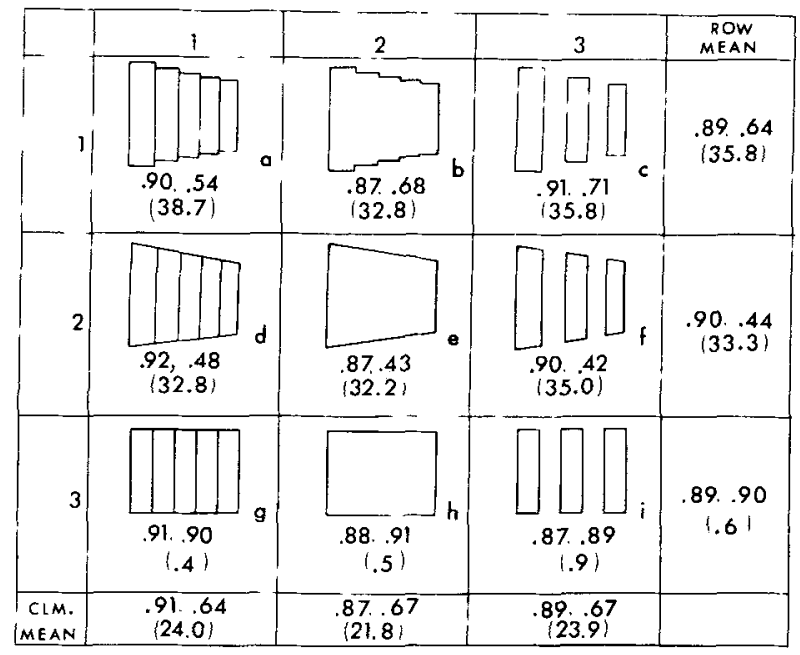

Figure 4. Stimuli and results for Experiments 3 and 4. Results are shown as $T 1, T 2$ (S), where $T 1$ and $T 2$ are the mean proportions of time that direction of rotation was judged correctly for Phase I and Phase II (Experiment 3 ) and $S$ is the mean judged slant (Experiment 4). 
Stimuli. The stimuli were nine plane outline forms rotating about a vertical axis at the center of the form. The forms were based on the same slanted outline rectangle as in Experiment 1. The projection of the slanted rectangle is shown as form e in Figure 4. Five smaller frontally oriented rectangles were arranged at equal distances along the slanted rectangle, as in Experiment 1, but, unlike the frontally oriented rectangles in Experiment 1, these five rectangles did not overlap horizontally. The projection of the five rectangles is shown as form a. Form $d$ is the projection of the slanted outline rectangle to which vertical lines have been added to produce a horizontal perspective gradient matching that in form a. Form $\mathrm{g}$ is an equally divided rectangle. The forms in the middle column display the same outlines as those on the left. The forms in the column on the right were produced by deleting alternate segments from the forms on the left. The overall form dimensions, projection distances, number of rotations, rotation speed, and ISI were the same as in Experiment 1.

Apparatus and Procedure. The apparatus and procedure were the same as in Experiment 1, except that there were 45 regular stimuli.

\section{Results and Discussion}

As in Experiment 1, the proportion of veridical judgment time was analyzed for each stimulus, for the second $360 \mathrm{deg}$ of rotation. This $360 \mathrm{deg}$ again was divided into two 180-deg phases, with the period during which the left side of the form (as shown in Figure 4) was closer labeled as Phase I and the period during which the left side was more distant labeled as Phase II. For the trapezoidal shapes, Phase I is the veridical phase and Phase II is the illusory phase. The mean proportions of veridical judgment time are shown in Figure 4. Separate four-way analyses of variance (continuity, shape, perspective, and subjects) were conducted for Phase I and Phase II.

Phase I. The main effect of perspective $[F(4,116=$ 40.5] and the interaction of perspective with shape $[F(8,232)=5.6]$ were significant $(p<.01)$. The main effects of continuity and shape and the remaining interactions were not significant. As expected, the proportion of veridical judgment time increased with decreasing projection point distance, with the increase more rapid for the forms that were rectangular in overall shape.

Phase II. Significant main effects were found for shape $[F(2,58)=158.5, p<.01]$ and for perspective $[F(4,116)=187.6, p<.01]$, but not for continuity $[F(2,58)=2.30, p>.05]$. The interactions of shape with continuity $[F(4,116)=9.6]$ and with perspective $[F(8,232)=28.5]$ were significant $(p<.01)$ (see Figure 5). The remaining interactions were not significant. Post hoc comparisons (Tukey HSD test) were conducted among the three shape levels at each continuity level. For the div ded forms with continuous external contours (column 1 in Figure 4), the proportion of veridical response time was significantly greater for the rectangle $(\mathrm{g})$ than for either trapezoidal shape (a and d). The trapezoidal shapes did not differ significantly. In Experimer: 1, a significant difference was found for similar fcrms (b vs. $c$ and e vs. $f$ in Figure 2). There is no obvious explanation for the

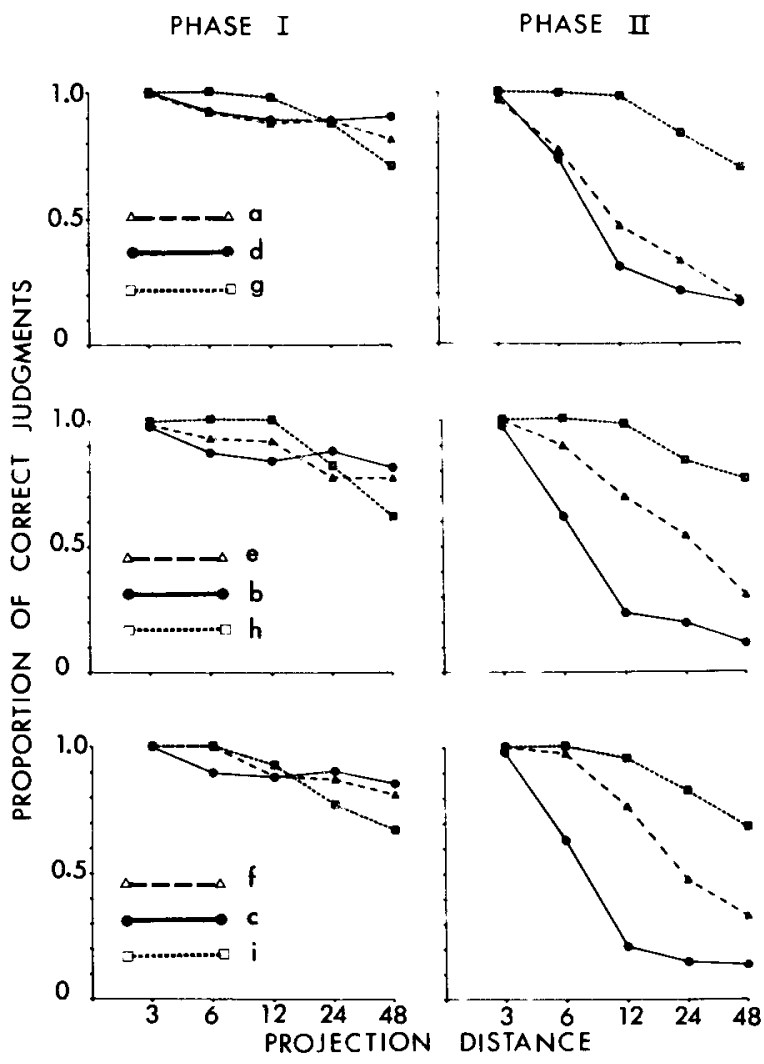

Figure 5. Shape by perspective interactions for each continuity level, for Phase I (left) and Phase II (right). (Small letters refer to forms in Figure 4.)

smaller difference found between the two forms in Experiment 3 as compared to Experiment 1. Although the horizontal gradient is different in the Experiment 3 forms, this gradient is intermediate between the row 1 and row 2 gradients in Experiment 1 . For the figures that are not divided internally (column 2 in Figure 4) and those lacking continuous external contours (column 3), all pairs of shape levels differed significantly. The proportion of veridical response time was highest for the rectangular shapes (h and $i$ ), next highest for the trapezoidal shapes with explicit right angles (b and c), and lowest for the trapezoidal shapes that did not show right angles (e and $f$ ). Overall, the presence or absence of internal subdivisions in a form or of a continuous perimeter did not consistently affect the proportion of veridical judgment time.

There was an increase in the proportion of veridical response time when right angles were present in forms showing a relative size gradient, whether the form consisted of an undivided outline (b vs. e) or separate segments (c vs. f). This confirms the finding in Experiment 1 that, for a given relative size gradient, the presence of right angles increases the veridicality of direction of rotation judgments. 


\section{EXPERIMENT 4}

The purpose of Experiment 4 was to collect static slant judgments for the nine forms studied in Experiment 3 .

\section{Method}

Subjects. The subjects were 18 paid university students. The vision criterion was the same as in Experiment 1.

Stimuli. The stimuli were $35-\mathrm{mm}$ black-and-white slides of the nine forms used in Experiment 3.

Apparatus and Procedure. The apparatus and procedure were the same as in Experiment 2, except that a total of 31 slides were presented, consisting of four practice slides and three presentations of each of the nine forms.

\section{Results and Discussion}

Mean judged slants for the nine stimuli are shown in Figure 4. The main effect of shape was significant $[F(2,34)=254.2, p<.01]$. Post hoc comparisons (Tukey HSD test) showed significantly lower judged slant for the rectangular shapes (row 3 in Figure 4) than for the trapezoidal shapes, both with and without right angles (rows 1 and 2). The forms in row 2 did not differ significantly from those in row 1 in judged slant. The main effect of continuity $[F(2,34)=2.07]$ and the interaction of shape and continuity $[F(4,68)=2.01]$ were not significant $(p>.05)$. The lack of a significant continuity effect meant that the predicted difference in perceived relative distance among the forms in rows 1 and 2 was not confirmed (although five of the six pairwise comparisons among these forms were in the predicted direction).

If the differences in proportion of veridical judgment time for direction of rotation found in Experiment 3 between forms $b$ and $c$ and between forms $e$ and $f$ in Figure 4 were due to misperceived static slant, form b should have been judged as less slanted than $e$, and $c$ as less slanted than $\mathrm{f}$. Instead, the mean judged slants were almost identical for each of these pairs of forms. (The small differences that occurred were in the direction opposite to that predicted by a misperceived static slant explanation of misperceived direction of rotation.) These results confirm those obtained in Experiments 1 and 2: Misperceived direction of rotation cannot be predicted from misperceived static slant when the presence of right angles in the stimulus forms is varied.

\section{GENERAL DISCUSSION}

If relative size gradients determined the veridicality of the direction of rotation judgments, then equal levels of veridicality should have occurred for the graded-rectangle forms and the trapezoids displaying the same gradients. If, on the other hand, changes in the projected contour angles determined the veridicality of these judgments, the graded-rectangle forms should have produced the same high level of veridicality as the rectangles. The results of Experiments 1 and 3 were clearly intermediate between these two possibilities. Direction of rotation judgments for the graded-rectangle forms were more accurate than those obtained with trapezoids but less accurate than those obtained with rectangles.

The following explanation is suggested for these intermediate results: Direction judgments are affected both by changes in the explicit contour angles and by changes in contour angles implicit in the overall shape of the form. In the case of simple outline trapezoids and rectangles, the explicit and implicit contour angles are the same. In the case of the forms displaying a size gradient by means of rectangular segments, the explicit contour angles are right angles, but the overall shape is trapezoidal. Imaginary lines connecting the vertical contours would form acute angles enclosing the larger contour and obtuse angles enclosing the smaller contour. Judged direction of rotation for these forms appears to have been a compromise between the direction suggested by changes in the explicit contour angles and that suggested by changes in the implicit angles. For example, at an intermediate perspective level with the large side of a graded-rectangle form receding, the right angles enclosing that side became increasingly obtuse, but the implicit angles formed by connecting the endpoints of this side with the small vertical side became increasingly acute. If the large side was moving from left to center, the explicit angle change would have implied a clockwise rotation, but the overall shape change would have implied a counterclockwise rotation.

The difference between the graded-rectangle forms and the trapezoids in veridicality of direction judgments cannot be attributed to the rate or magnitude of changes in the visual angles subtended by the vertical edges of the forms during rotation, as these functions were identical for both types of forms. This is because the vertical edges and their separations were identical for all forms displaying a vertical perspective gradient.

The possibility that the veridicality of direction judgments is determined by misperceived static slant was not supported when veridicality was varied by varying the presence of right angles in the forms. The presence of right angles did not significantly affect static slant judgments for forms displaying the same relative size gradients. (The nonsignificant differences that did occur were in the direction predicted by the misperceived slant explanation in only one of five comparisons.) These results do not contradict earlier findings (Olson, 1974; Power \& Day, 1973) of high correlations between slant judgments and measures of apparent reversal of direction of rotation of plane objects. These correlations were found across 
variations in linear perspective produced by variations in the slopes of the horizontal contours of outline trapezoids (Olson, 1974) or in the slopes of horizontal lines affixed to ellipses (Power \& Day, 1973). The present results demonstrate that another stimulus variable - the presence or absence of right anglescan alter the veridicality of direction judgments without altering judged static slant. This implies that misperceptions of direction of rotary motion cannot be completely explained on the basis of figural characteristics that alter perceived static slant.

The use of contour interruptions to suggest interposition increased the proportion of veridical direction judgments for some stimuli and decreased that proportion for others. The increase occurred for forms that placed interposition in conflict with a relative size gradient in the vertical dimension, that is, the contours of the larger segments were interrupted by the contours of the smaller segments (forms $a$ and $d$ in Figure 2). Veridical judgment time increased when the larger side was more distant in the displayed rotation (the illusory phase of the rotating trapezoid illusion). Conflicting interposition information thus reduced the effectiveness of the trapezoid illusion.

The decrease in veridical judgment time occurred for forms that did not display a vertical size gradient (forms $g$ and $j$ in Figure 2), when the more distant segments interrupted the contours of the closer segments. This can be regarded as a new illusion of misperceived direction of rotary motion, based entirely on false interposition.

False interposition is a static, rather than a dynamically changing, characteristic of a rotating figure. The relative distances of segments implied by contour interruption is fixed during rotation, as contrasted to the projected contour angles, which vary during rotation. The effectiveness of false interposition in determining the veridicality of direction judgments demonstrates that static indicators of relative distance, as well as dynamic indicators of changing relative distance, can play a role in direction of rotation judgments. For the specific contour interruptions and the size gradient used in Experiment 1, the size gradient was more effective in determining direction judgments. The size gradient could undoubtedly be made so shallow that it would be less effective than interposition, but, in considering the possible ranges of these two variables, it seems likely that a size gradient would be generally more effective than static interposition in determining direction judgments, especially if the contour angles produced by this gradient were explicitly displayed.

In summary, three conclusions may be reached from the results of these experiments: (1) The explicitly displayed contour angles affect the perception of direction of rotary motion even when perspective gradients are held constant. The proportion of veridical judgments increases in the presence of right angles. (2) The effect of the presence or absence of right angles is not due to variations in perceived static slant. (3) Perceived direction of rotation can be affected by a static false interposition cue, with interposition increasing the proportion of veridical judgments when placed in conflict with a relative size gradient and decreasing this proportion when added to a figure lacking a size gradient. These results generally support an explanation of the perception of direction of rotation based primarily on dynamic factors (proximal contour angle changes), but also demonstrate that a static factor (interposition) can affect these judgments.

\section{REFERENCES}

Ames, A. Visual perception and the rotating trapezoidal window. Psychological Monographs, 1951, 65(7, Whole No. 324).

BörJesson, E. Properties of changing patterns evoking visually perceived oscillation. Perception \& Psychophysics, 1971, 9, 303-308

Braden, J. H. The effect of varying linear perspective, movement parallax, and speed of rotation on perceived oscillation of twodimensional trapezoidal stimuli. Perception \& Psychophysics, 1978, 23, 51-57.

Braunste in, M. L. Perception of rotation in figures with rectangular and trapezoidal features. Journal of Experimental Psychology, 1971, 91, 25-29.

Braunstein, M. L. Perception of rotation in depth: A process model. Psychological Review, 1972, 79, 510-524.

Braunste in, M. L. Perceived direction of rotation of simulated three-dimensional patterns. Perception \& Psychophysics, 1977, 21, 553-557.

Braunstein, M. L., \& Payne, J. W. Perspective and the rotating trapezoid. Journal of the Optical Society of America, 1968, 58, 399-403.

Cross, J., \& Cross, J. The misperception of rotary motion. Perception \& Psychophysics, 1969, 5, 94-96.

GOGEL, W. C. The adjacency principle and three-dimensional visual illusions. Psychonomic Monograph Supplement, 1970, 3(Whole No. 45).

Hershberger, W. A., Stewart, M. R., \& Laughlin, N. K. Conflicting motion perspective simulating simultaneous clockwise and counterclockwise rotation in depth. Journal of Experimental Psychology: Human Perception and Performance, 1976, 2, 174-178.

LEE, W. Experimental design and analysis. San Francisco: Freeman, 1975.

OLson, R. K. Slant judgments from static and rotating trapezoids correspond to rules of perspective geometry. Perception \& Psychophysics, 1974, 15, 509-516.

Petersik, J. T. Three-dimensional object constancy: Coherence of a simulated rotating sphere in noise. Perception \& Psychophysics, 1979, 25, 328-335.

Power, R. P., \& DAY, R. H. Constancy and illusion of apparent direction of rotary motion in depth: Tests of a theory. Perception \& Psychophysics, 1973, 13, 217-223.

(Received for publication October 10, 1979; revision accepted January 23, 1980.) 